\title{
Biochanin A attenuates LPS-induced pro-inflammatory responses and inhibits the activation of the MAPK pathway in BV2 microglial cells
}

\author{
WANG-YANG WU ${ }^{1,2^{*}}$, YANG-YANG WU ${ }^{1,2^{*}}$, HUAN HUANG ${ }^{1}$, CAN HE $^{1}$, WEI-ZU LI ${ }^{1}$, \\ HUI-LI WANG ${ }^{2}$, HAN-QING $\mathrm{CHEN}^{2}$ and YAN-YAN YIN ${ }^{1}$ \\ ${ }^{1}$ Department of Pharmacology, Anhui Medical University, Hefei, Anhui 230032; ${ }^{2}$ School of Biotechnology \\ and Food Engineering, Hefei University of Technology, Hefei, Anhui 230009, P.R. China
}

Received May 31, 2014; Accepted November 11, 2014

DOI: $10.3892 / \mathrm{ijmm} .2014 .2020$

\begin{abstract}
Inflammation in the brain, characterized by the activation of microglia, is believed to participate in the pathogenesis of Parkinson's disease. Biochanin A, an $O$-methylated isoflavone, is a natural organic compound and is classified as a phytoestrogen. In this study, using murine BV2 microglial cells, we investigated the anti-inflammatory effects of biochanin A and the possible mechanisms involved. BV2 microglial cells were treated with lipopolysaccharide (LPS) to induce pro-inflammatory responses and the cells were then treated with biochanin A. Cell viability was examined by MTT assay. The production of nitric oxide (NO) was examined using Griess reagent and intracellular reactive oxygen species (ROS production) was measured by DCFH-DA assay. The mRNA expression of interleukin-1 $\beta$ (IL-1 $\beta$ ), inducible nitric oxide synthase (iNOS) and tumor necrosis factor- $\alpha$ (TNF- $\alpha$ ) was examined by RT-PCR. The expression of p-ERK, p-JNK, p-p38 and iNOS was measured by western blot analysis. In addition, the protein and mRNA and phosphorylation levels of pro-inflammatory cytokines were determined by western blot analysis and RT-PCR, respectively. The results revealed that biochanin A attenuated LPS-induced microglial activation and the production of TNF- $\alpha$, IL-1 $\beta$, nitric oxide and reactive oxygen species in a dose-dependent manner. Biochanin A significantly decreased the LPS-induced mRNA expression of TNF- $\alpha$ and IL-1 $\beta$, and inhibited iNOS mRNA and protein
\end{abstract}

Correspondence to: Professor Han-Qing Chen, School of Biotechnology and Food Engineering, Hefei University of Technology, 193 Tunxi Road, Hefei, Anhui 230009, P.R. China

E-mail: hanqchen@hfut.edu.cn

Professor Yan-Yan Yin, Department of Pharmacology, Anhui Medical University, 81 Meishan Road, Hefei, Anhui 230032, P.R. China

E-mail: yinyanyan5678@126.com

*Contributed equally

Key words: biochanin A, proinflammatory cytokines, reactive oxygen species, nitric oxide, mitogen-activated protein kinases, BV2 microglial cells expression. Furthermore, biochanin A significantly inhibited the LPS-induced phosphorylation of c-Jun NH2-terminal kinase (JNK), extracellular signal-regulated kinase (ERK) and p38. These findings suggest that the inhibitory effects of biochanin A on LPS-induced proinflammatory responses may be associated with the inhibition of mitogen-activated protein kinase (MAPK) signaling pathways in BV2 microglial cells.

\section{Introduction}

Inflammation in the brain, characterized by the activation of microglia, has been closely associated with the pathogenesis of Parkinson's disease (PD), as well as several other neurodegenerative disorders, including Alzheimer's disease $(1,2)$. Microglia, the resident immune cells in the brain, play a role in immune surveillance and host defense under normal conditions (3). However, in response to injury, infection, or inflammation, microglia become readily activated and secrete a variety of proinflammatory factors, including cytokines such as tumor necrosis factor- $\alpha(\mathrm{TNF}-\alpha)$ and interleukin- $1 \beta$ (IL-1 $\beta)$, as well as the free radicals, nitric oxide (NO) and reactive oxygen species (ROS). The accumulation of these proinflammatory factors is considered to contribute to the degeneration of dopaminergic neurons (4-8). As the midbrain region that encompasses the substantia nigra is particularly rich in microglia (6), the activation of microglia and the release of proinflammatory factors may be a crucial component of the degenerative process of dopaminergic neurons in PD. Hence, the identification of compounds that inhibit microglial activation and the release of proinflammatory factors is highly desirable in the search for therapeutic agents for inflammation-mediated neurodegenerative diseases, including PD.

In the investigation of more potent anti-inflammatory agents, natural compounds have received much attention in recent years $(9,10)$. Biochanin A, an $O$-methylated isoflavone, is a natural organic compound and is classified as a phytoestrogen, due to its structural similarity to estrogen and its estrogen-like activities. It can be found predominantly in legume plants, such as chickpeas and red clover. A number of studies have demonstrated that biochanin A exerts beneficial effects on human health, including the prevention of cancers, heart 
disease, menopausal symptoms and osteoporosis $(11,12,13)$. In our previous study, we found that biochanin A inhibited the lipopolysaccharide (LPS)-induced activation of microglia and the production of TNF- $\alpha$, NO and superoxide in mesencephalic neuron-glia cultures and microglia-enriched cultures (11). However, the mechanisms underlying the anti-inflammatory effects of biochanin A are not yet fully understood.

In the present study, using murine BV2 microglial cell cultures, we further investigated the potential anti-inflammatory effects of biochanin $\mathrm{A}$, as well as the possible mechanisms involved.

\section{Materials and methods}

Materials. All reagents used for cell culture were purchased from Gibco(Carlsbad,CA,USA). Biochanin A,LPS and3-(4,5-dimethylthiazol-2-yl)-2,5-diphenyltetrazolium bromide (MTT) were purchased from Sigma-Aldrich (St. Louis, MO, USA). Griess reagent and TRIzol RNA extraction reagent were provided by the Beyotime Institute of Biotechnology (Haimen, China). RT-PCR reagents were purchased from Thermo Scientific (Waltham, MA, USA). Primary antibody specific for inducible NO synthase (iNOS; 2982s) was obtained from Cell Signaling Technology (Danvers, MA, USA). Primary antibodies against extracellular signal-regulated kinase (ERK; BS6426), phosphorylated (p)-ERK (AP0484), c-Jun NH2-terminal kinase (JNK; BS6706), p-JNK (BS4763), p38 (BS3566) and p-p38 (BS4635) were obtained from Bioworld Technology (St. Louis Park, MN, USA). A secondary antibody for goat anti-rabbit immunoglobulin ( $\operatorname{IgG})$ horseradish peroxidase (HRP) was acquired from Zhongshan Biotechnology Corp. (Beijing, China). The TNF- $\alpha$ and IL-1 $\beta$ ELISA kits were obtained from BD Biosciences (San Jose, CA, USA). Other chemicals and reagents used in this study were of analytical grade.

Cell culture and MTT assay. The BV-2 microglial cell line was obtained from Shanghai Fuxiang Biological Corp. (Shanghai, China). The cells were seeded in 96-well culture plates at $1 \times 10^{5}$ cells/well. The cell cultures were maintained at $37^{\circ} \mathrm{C}$ in a humidified atmosphere of $5 \% \mathrm{CO}_{2}$ and $95 \%$ air in Dulbecco's modified Eagle's medium/nutrient F12 (DMEM/F12) containing $10 \%$ fetal bovine serum (FBS) and antibiotics (100 U/ml penicillin and $100 \mu \mathrm{g} / \mathrm{ml}$ streptomycin). For cell viability assay, the cells were incubated with serum-free medium for $24 \mathrm{~h}$, followed by treatment with various concentrations of LPS or biochanin A. Following incubation for $36 \mathrm{~h}, 100 \mu \mathrm{l}$ of MTT $(0.5 \mathrm{mg} / \mathrm{ml}$ final concentration) were added and incubation was continued for a further $4 \mathrm{~h}$. The formazan crystals in each well were dissolved in dimethyl sulfoxide (DMSO), and the absorbance was measured at $490 \mathrm{~nm}$ using an enzyme-linked immunosorbent assay (ELISA) microplate reader. The absorbance of the untreated control group was also measured in order to calculate relative cell viability.

Measurement of proinflammatory cytokine production. The production of proinflammatory cytokines was determined using an ELISA kit. BV2 cells were treated with various concentrations of biochanin A and stimulated with LPS for $36 \mathrm{~h}$. The culture medium was collected and the concentrations of TNF- $\alpha$ and IL-1 $\beta$ were measured according to the manufacturer's instructions with quantikine mouse TNF- $\alpha$ and IL-1 $\beta$ immunoassay.
Determination of $\mathrm{NO}$ production. The BV2 cells were pretreated with various concentrations of biochanin A (1.25, $2.5,5 \mu \mathrm{M})$ for $30 \mathrm{~min}$ and then treated with LPS $(10 \mu \mathrm{g} / \mathrm{ml})$ for an additional $36 \mathrm{~h}$. The production of NO was measured by Griess reaction as previously described (14). Briefly, after $36 \mathrm{~h}$ of treatment with LPS $(10 \mu \mathrm{g} / \mathrm{ml})$ and various concentrations of biochanin A, $50 \mu \mathrm{l}$ of culture supernatant from each sample were mixed with an equal volume of Griess reagent [1\% sulfanilamide/0.1\% N-(1-naphthyl)-ethylenediamine dihydrochloride $/ 2.5 \%$ phosphoric acid]. Following incubation for $15 \mathrm{~min}$, the absorbance values were read at $540 \mathrm{~nm}$ using an ELISA microplate reader. The nitrite content was calculated compared with that of standard concentrations of sodium nitrite dissolved in DMEM.

Measurement of intracellular ROS by 2',7'-dichlorofluorescein diacetate (DCFH-DA) assay. The intracellular formation of ROS was assessed using the non-fluorescent probe, DCFH-DA. DCFH-DA passively diffuses into cells and is deacetylated by esterases to form non-fluorescent DCFH. DCFH reacts with ROS to form the fluorescent product, DCF, which is trapped inside the cells. The BV2 cells were seeded at a density of $1 \times 10^{5}$ cells/well in a 96-plate and pre-treated with various concentrations of biochanin A for $30 \mathrm{~min}$, then treated with LPS $(10 \mu \mathrm{g} / \mathrm{ml})$ for an additional $36 \mathrm{~h}$. The culture medium was first removed and the cells were washed with PBS 3 times. DCFH$\mathrm{DA}$, diluted to a final concentration of $10 \mu \mathrm{M}$ with DMEM/ F12, was added to the culture medium and incubated at $37^{\circ} \mathrm{C}$ for $20 \mathrm{~min}$ in the dark. After washing the cells 3 times with serum-free medium, the cells were visualized using an inverted fluorescence microscope [Olympus Opticals, Tokyo, Japan; excitation (Ex)/emission (Em), 352/461 nm]. Six continual fields in each group were used for quantitative analysis. By using the Image-Pro Plus 6.0 analysis system, the fluorescence intensity in each group was measured to indicate the production of ROS.

RNA extraction and reverse transcription-polymerase chain reaction $(R T-P C R)$. The $\mathrm{BV} 2$ cells $\left(16 \times 10^{5}\right.$ cells/well in 6-plate) were treated with LPS in the presence or absence of biochanin A, and total RNA was extracted using TRIzol reagent according to the manufacturer's instructions. The yield and purity of the RNA preparations were examined spectrophotometrically at $260 \mathrm{~nm}$ and $280 \mathrm{~nm}$, respectively. An equal amount of RNA $(2 \mu \mathrm{g})$ was used for each cDNA synthesis reaction using a reverse transcription system. The following primers derived from the published cDNA sequences were used for the PCR amplifications: IL-1 $\beta$ forward, 5'-CTC CAT GAG CTT TGT ACA AGG-3' and reverse, 5'-TGC TGA TGT ACC AGT TGG GG-3'; iNOS forward, 5'-CCC TTC CGA AGT TTC TGG CAG CAG C-3' and reverse, 5'-GGC TGT CAG AGC CTC GTG GCT TTG G-3'; TNF- $\alpha$ forward, 5'-TTC TGT CTA CTG AAC TTC GGG GTG ATC GGT CC-3' and reverse, 5'-GTA TGA GAT AGC AAA TCG GCT GAC GGT GTG GG-3'; GAPDH forward, 5'-GGT GAA GGT CGG TGT GAA CG-3' and reverse, 5'-TTG GCT CCA CCC TTC AAG TG-3'. The products were inspected visually on a $1 \%$ precast agarose gel with ethidium bromide staining and the bands were quantified by densitometry. Ratios were calculated for the IL-1 $\beta$, iNOS and TNF- $\alpha$ signals with the control signals from GAPDH. The averages from these ratios were presented. 
Table I. Effects of biochanin A on pro-inflammatory cytokine production in LPS-stimulated BV2 microglial cells.

\begin{tabular}{lrr}
\hline & \multicolumn{1}{c}{ Pro-inflammatory cytokine concentration } \\
\cline { 2 - 3 } Groups & TNF- $\alpha(\mathrm{pg} / \mathrm{ml})$ & $\mathrm{IL}-1 \beta(\mathrm{pg} / \mathrm{ml})$ \\
\hline Control & $107.82 \pm 18.14$ & $54.12 \pm 9.413$ \\
LPS $(10 \mu \mathrm{g} / \mathrm{ml})$ & $2269.75 \pm 62.38^{\mathrm{a}}$ & $213.12 \pm 15.64^{\mathrm{a}}$ \\
Biochanin A $(1.25 \mu \mathrm{M})+$ LPS & $2037.37 \pm 66.56^{\mathrm{b}}$ & $203.65 \pm 14.13$ \\
Biochanin A $(2.5 \mu \mathrm{M})+$ LPS & $1776.62 \pm 47.71^{\mathrm{c}}$ & $170.80 \pm 13.34^{\mathrm{b}}$ \\
Biochanin A $(5 \mu \mathrm{M})+$ LPS & $1577.67 \pm 50.84^{\mathrm{c}}$ & $147.09 \pm 14.36^{\mathrm{b}}$ \\
\hline
\end{tabular}

Results are the means \pm SD of at least 3 independent experiments. ${ }^{\mathrm{a}} \mathrm{P}<0.01$ compared with the control (untreated) group, ${ }^{\mathrm{b}} \mathrm{P}<0.05$, ${ }^{\mathrm{c}} \mathrm{P}<0.01$ compared with the lipopolysaccharide (LPS)-treated group.

Western blot analysis. Following treatment, the BV2 cells were rinsed in ice-cold PBS and lysed in RIPA buffer (Invitrogen, Amersham, UK). The protein concentration was determined by bicinchoninic acid (BCA) assay (Wuhan Boster Biological Technology, Ltd., Wuhan, China). The extracted protein samples were separated by $12 \%$ SDS-polyacrylamide gel electrophoresis (SDS-PAGE) and transferred onto polyvinylidene difluoride (PVDF) membranes (Millipore Corp., Billerica, MA, USA). The membranes were incubated with blocking solution (5\% skim milk) to block non-specific protein binding, followed by incubation with the following primary antibodies at $4^{\circ} \mathrm{C}$ overnight: $\beta$-actin, p-JNK, JNK, p-ERK, ERK, p-p38, p38, iNOS. The membranes were incubated with a horseradish peroxidase (HRP)-conjugated secondary antibody at room temperature for $2 \mathrm{~h}$, and then specific protein bands were detected using the ECL-chemiluminescence kit (Thermo Scientific) according to the manufacturer's instructions. The immunoblot bands were visualized using a Bioshine ChemiQ 4600 mini Chemiluminescence imaging system (Bioshine, Shanghai, China) and protein expression was quantified using ImageJ software (National Institutes of Health, Bethesda, MD, USA).

Statistical analysis. The data are expressed as the means \pm SD $(n=3)$. Statistical significance was assessed by one-way ANOVA, Duncan's multiple range tests were used to determine significant differences between groups. A value of $\mathrm{p}<0.05$ was considered to indicate a statistically significant difference.

\section{Results}

Cytotoxic effects of biochanin A and LPS on BV2 microglial cells. To exclude the cytotoxicity caused by biochanin A treatment, the BV2 microglial cells were treated with biochanin A at concentrations of $1.25-30 \mu \mathrm{M}$ for $36 \mathrm{~h}$. The viability of the BV2 microglial cells was measured by MTT assay. At the examined concentrations of $1.25-5 \mu \mathrm{M}$ for $36 \mathrm{~h}$, biochanin $\mathrm{A}$ did not affect cell viability (Fig. 1A). However, treatment with concentrations $>5 \mu \mathrm{M}$ of biochanin A decreased cell viability. Thus, we selected the dose of $1.25-5 \mu \mathrm{M}$ of biochanin $\mathrm{A}$ for further experiments.

Furthermore, as we used LPS to induce microglial inflammatory responses, it was necessary to ascertain the non-toxic concentration of LPS in BV2 cells. Treatment with
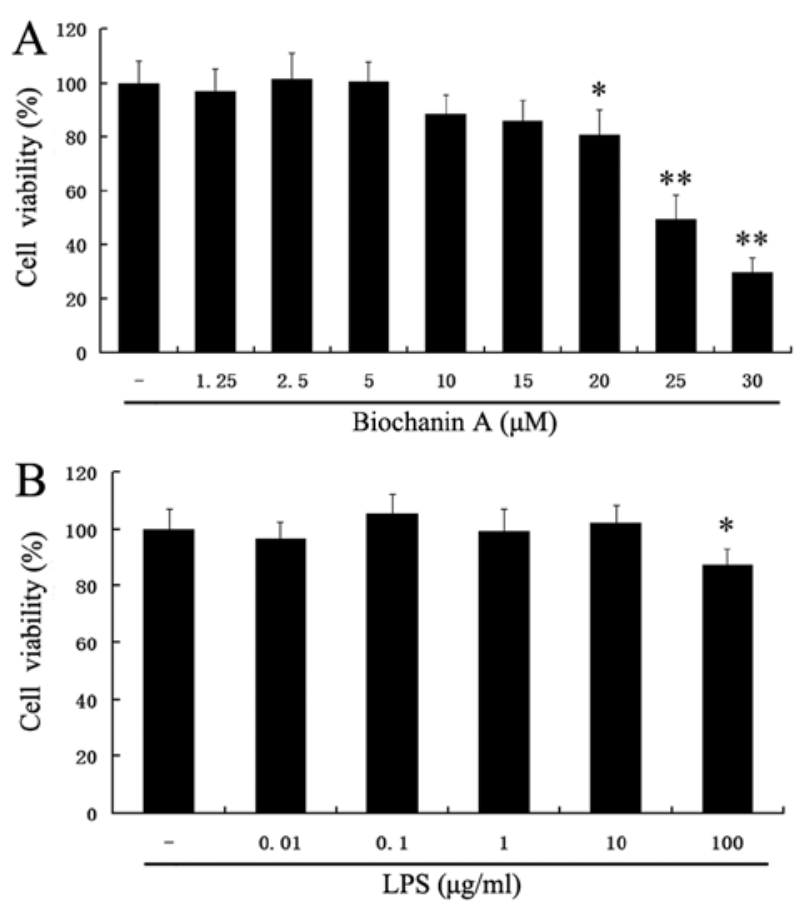

Figure 1. Cell viability of BV2 cells. (A) Cytotoxicity of biochanin A in BV2 cells was assessed by MTT assay. (B) Following treatment with various concentration of lipopolysaccharide (LPS) for $36 \mathrm{~h}$, the viability of the BV2 cells was assessed by MTT assay. Values correspond to the means of 3 independent experiments and are shown as the percentage of viable cells compared with the viability of untreated cells. ${ }^{*} \mathrm{P}<0.05,{ }^{* *} \mathrm{P}<0.01$ compared with the untreated cells.

LPS $(0.01-10 \mu \mathrm{g} / \mathrm{ml})$ did not affect the cell viability $(\mathrm{p}>0.05)$ (Fig. 1B). In addition, our preliminary experimental results suggested that $10 \mu \mathrm{g} / \mathrm{ml}$ LPS markedly induced proinflammatory cytokine production (data not shown). Therefore, we selected the dose of $10 \mu \mathrm{g} / \mathrm{ml}$ of LPS for further experiments.

Effects of biochanin A on LPS-induced morphological changes in BV2 cells. We also observed the morphological changes occurring in microglial activation following treatment with LPS. As shown in Fig. 2, the microglia exhibited the typical ramified morphology of resting microglia in the control group. Following treatment with LPS $(10 \mu \mathrm{g} / \mathrm{ml} ; 36 \mathrm{~h})$, the microglia became activated with a greatly enlarged cell body and the 

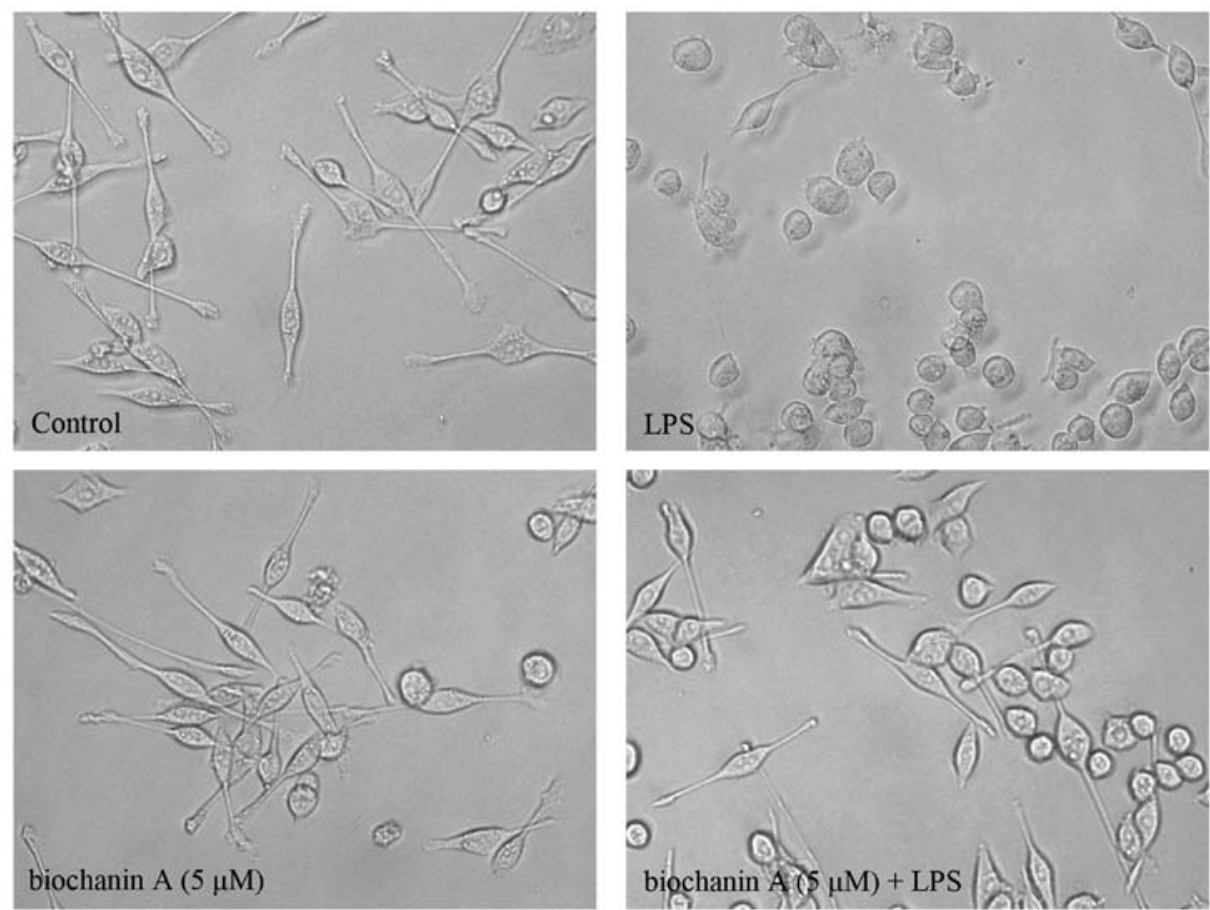

Figure 2. Effects of biochanin A on lipopolysaccharide (LPS)-induced morphological changes in BV-2 cells. BV-2 cells were treated for 30 min with the vehicle (DMSO) or the indicated concentrations of biochanin A prior to treatment with $10 \mu \mathrm{g} / \mathrm{ml}$ LPS for $36 \mathrm{~h}$. Images presented are from one experiment and representative of at least 3 independent experiments.

A

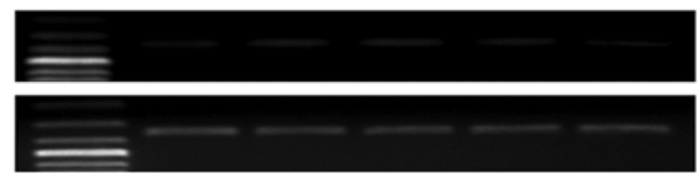

TNF- 0

GAPDH

$$
\text { 咅 }
$$$$
\text { 휴 }
$$

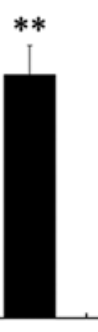

$+$



$+$

1.25

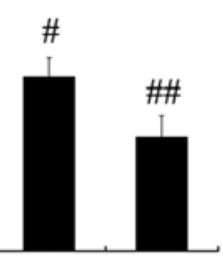

$+\quad+$

$2.5 \quad 5$
B


IL-1 $\beta$

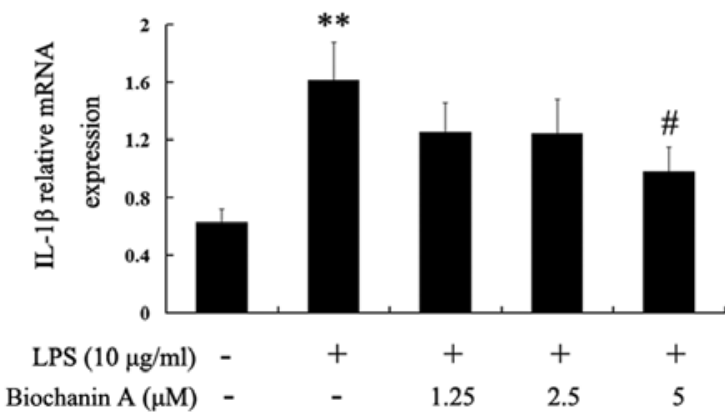

Figure 3. Inhibitory effects of biochanin A on (A) tumor necrosis factor- $\alpha$ (TNF- $\alpha$ ) and (B) interleukin-1 (IL-1 $\beta$ ) mRNA expression in BV2 cells. BV-2 cells were treated with or without biochanin A for $30 \mathrm{~min}$ prior to treatment with lipopolysaccharide (LPS; $10 \mu \mathrm{g} / \mathrm{ml}$ ). After $36 \mathrm{~h}$, the mRNA expression was analyzed by extraction and reverse transcription-polymerase chain reaction (RT-PCR). GAPDH was used as an internal control for PCR. Results are presented as the means \pm SD of at least 3 independent experiments. ${ }^{* *} \mathrm{P}<0.01$ compared with the control group; ${ }^{\#} \mathrm{P}<0.05$, ${ }^{\# \#} \mathrm{P}<0.01$ compared with the LPS-treated group.

characteristic shapes of activated microglia. However, pretreatment with biochanin A $(5 \mu \mathrm{M})$ significantly attenuated the LPS-induced microglial activation.

Effects of biochanin A on LPS-induced TNF- $\alpha$ and IL-1 $\beta$ production and $m R N A$ expression. Activated microglia are known to be a major source of various pro-inflammatory cytokines, such as TNF- $\alpha$ and IL-1 $\beta$ (15). In this study, we examined whether biochanin A reduces the generation of pro-inflammatory cytokines induced by LPS stimulation. As shown in Table I, the TNF- $\alpha$ and IL-1 $\beta$ levels were significantly increased in the culture medium of LPS-stimulated BV2 microglial cells. Pre-treatment with $1.25-5 \mu \mathrm{M}$ biochanin A significantly inhibited the production of TNF- $\alpha$ and IL- $1 \beta$ in a dose-dependent manner. To elucidate the mechanisms responsible for the inhibitory effects of biochanin A on TNF- $\alpha$ and IL- $1 \beta$ production, we examined the cytokine mRNA expression levels by RT-PCR. Consistent with the results obtained from cytokine production, the LPS-induced mRNA levels of TNF- $\alpha$ and IL-1 $\beta$ were reduced by biochanin A (Fig. 3), suggesting that biochanin A negatively regulated the production of TNF- $\alpha$ and IL- $1 \beta$ at the transcriptional level in the LPS-stimulated microglial cells. 
A
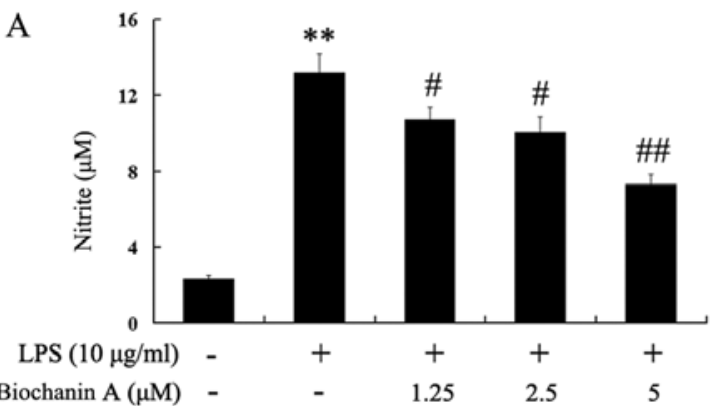

C
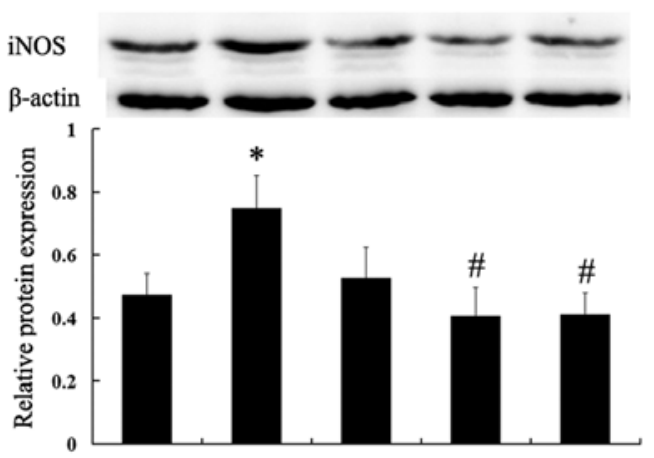

LPS $(10 \mu \mathrm{g} / \mathrm{ml})-$

Biochanin $\mathrm{A}(\mu \mathrm{M})$ -

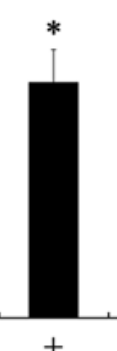

$+$

-

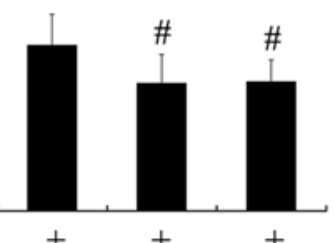

1.25
B
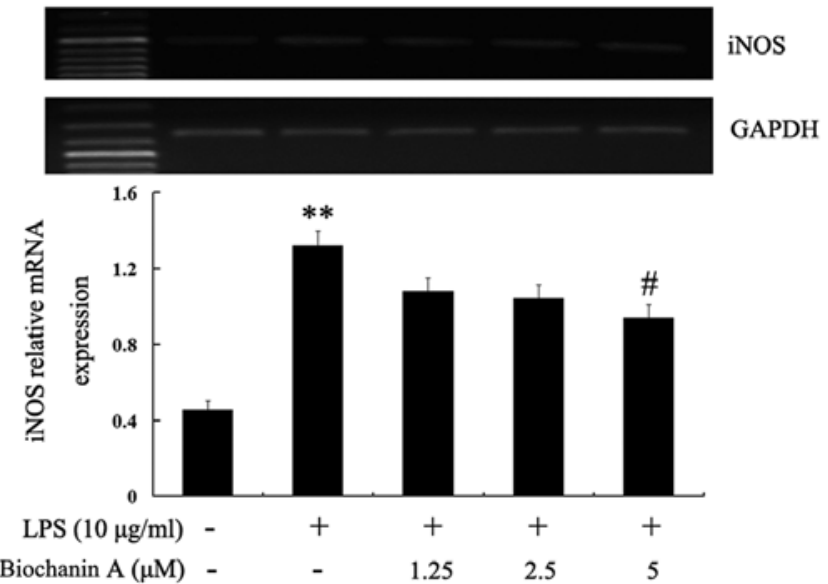

Figure 4. Inhibitory effects of biochanin A on nitric oxide (NO) production and inducible nitric oxide synthase (iNOS) expression in BV2 cells. Following pre-treatment with biochanin A for $30 \mathrm{~min}$, the BV2 cells were stimulated with lipopolysaccharide (LPS; $10 \mu \mathrm{g} / \mathrm{ml}$ ) and incubated for $36 \mathrm{~h}$. (A) The NO amounts were determined using Griess reagent. (B) In parallel experiments, the mRNA expression levels of iNOS were measured by reverse transcription-polymerase chain reaction (RT-PCR); and (C) the protein expression levels of iNOS were measured by western blot analysis. GAPDH was used as an internal control for PCR and $\beta$-actin was used for western blot analysis. The results are presented as the means \pm SD of at least 3 independent experiments. ${ }^{* *} \mathrm{P}<0.01$ compared with the control group; ${ }^{\#} \mathrm{P}<0.0,{ }^{\# \#} \mathrm{P}<0.01$ compared with the LPS-treated group.

Effects of biochanin A on the production of NO and the expression of iNOS. NO is generated from L-arginine by iNOS, and high levels of NO in the brain have been associated with the progression of various neurodegenerative diseases (16). In this study, to investigate the effects of biochanin A on NO production in LPS-stimulated BV2 cells, the BV2 cells were pre-treated with biochanin $\mathrm{A}(1.25-5 \mu \mathrm{M})$ for $30 \mathrm{~min}$ and then stimulated with LPS $(10 \mu \mathrm{g} / \mathrm{ml})$ for another $36 \mathrm{~h}$. The levels of nitrite in the culture medium were determined by Griess assay. The accumulation of nitrite in the culture supernatant was an indicator of the production of NO. Biochanin A significantly decreased the LPS-induced production of NO in the BV2 cells in a dose-dependent manner (Fig. 4A). Subsequently, to elucidate the mechanisms responsible for the inhibitory effects of biochanin A on NO production, we determined the mRNA and protein levels of iNOS by RT-PCR and western blot analysis, respectively. As shown in Fig. 4B and C, biochanin A effectively inhibited the mRNA and protein expression of iNOS in the LPS-stimulated BV2 cells. These results indicated that biochanin A inhibited NO production through the downregulation of the mRNA and protein expresion of iNOS in LPS-stimulated BV2 microglial cells.

Effects of biochanin A on intracellular ROS production. To determine whether the neuroprotective effects of biochanin A are due to a reduction in ROS production, the intracellular ROS production in BV2 cells was measured by DCFH-DA. As shown in Fig. 5, following treatment with LPS, the DCF fluorescence intensity was significantly increased. However, pre-treatment with biochanin A $(1.25-5 \mu \mathrm{M})$ attenuated the LPS-induced fluorescence intensity in the BV2 cells in a dose-dependent manner. These results confirm that biochanin A exerts a marked inhibitory effect on intracellular ROS production.

Effects of biochanin A on the phosphorylation of JNK, ERK and $p 38$. Mitogen-activated protein kinase (MAPK) signaling is known to be a major regulator of pro-inflammatory cytokines in LPS-stimulated microglia $(17,18)$. In order to investigate whether biochanin A inhibits the production of pro-inflammatory cytokines through the MAPK signaling pathway, we examined the effects of biochanin A on the LPS-induced phosphorylation of JNK, ERK and p38 in BV2 microglia by western blot analysis. As shown in Fig. 6, stimulation with LPS markedly increased the phosphorylation of JNK, ERK and p38. However, pre-treatment with biochanin $\mathrm{A}(5 \mu \mathrm{M})$ markedly inhibited the phosphorylation of JNK, ERK and p38. These results suggest that the phosphorylation of JNK, ERK and p38 is involved in the inhibitory effects of biochanin A on LPS-induced proinflammatory cytokine production in BV2 microglial cells.

\section{Discussion}

Biochanin A, an isoflavone compound, is found predominantly in chickpeas and red clover. In our previous study, we 
A
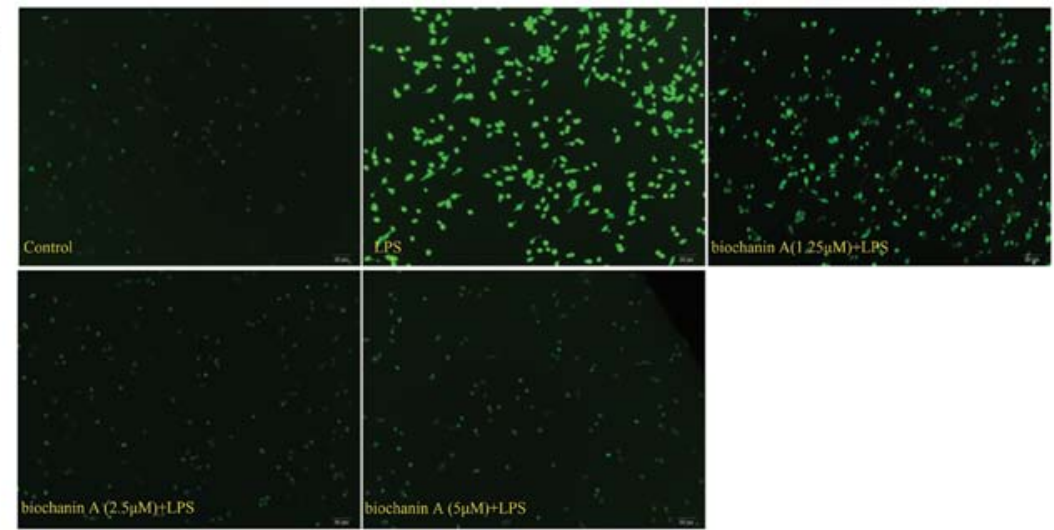

B

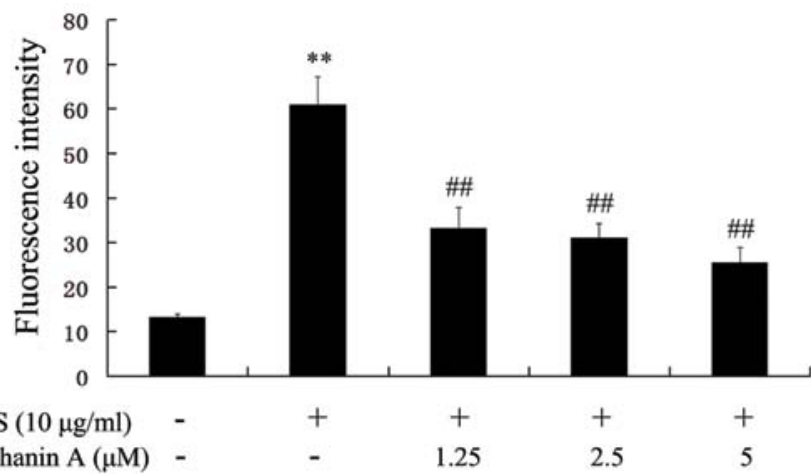

Figure 5. Effects of biochanin A on reactive oxygen species (ROS) production in BV2 cells. BV2 cells were pre-incubated with or without biochanin A or 30 min and then stimulated with lipopolysaccharide (LPS; $10 \mu \mathrm{g} / \mathrm{ml}$ ) for $36 \mathrm{~h}$. (A) Images presented are from one experiment and are representative of at least 3 independent experiments. (B) The fluorescence intensity is presented as the means $\pm \mathrm{SD}$ of at least 3 independent experiments. ${ }^{* *} \mathrm{P}<0.01$ compared with the control group; ${ }^{\#} \mathrm{P}<0.05,{ }^{\# \#} \mathrm{P}<0.01$ compared with LPS-treated group.

A

A p-JNK

JNK
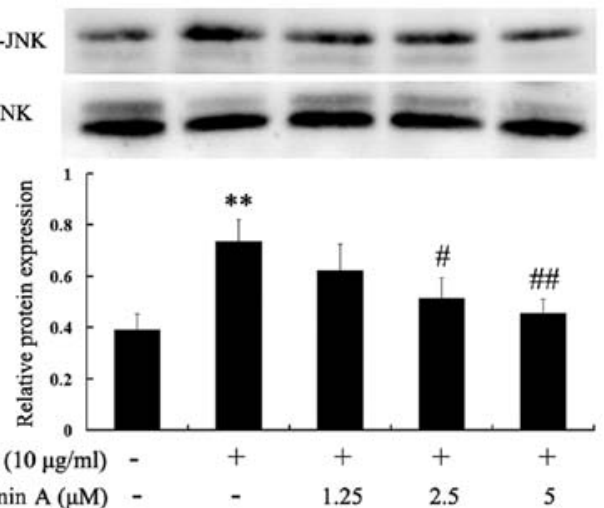

B

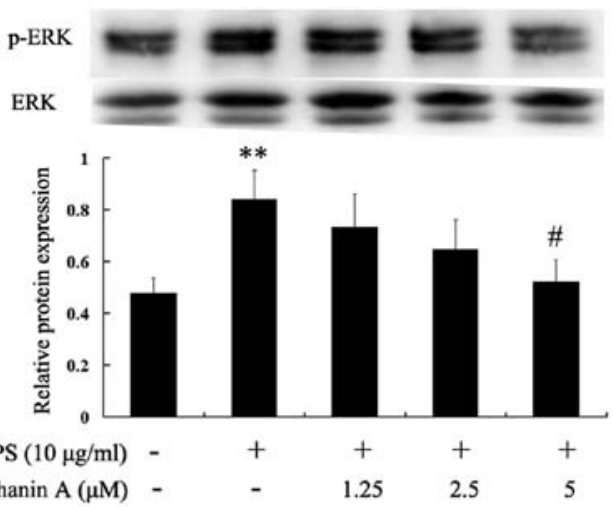

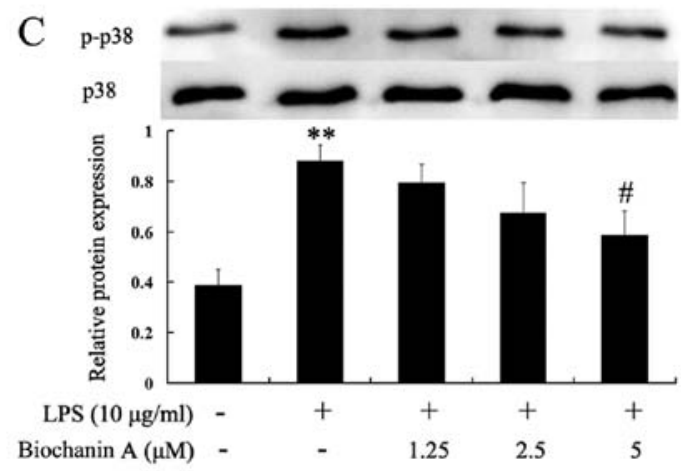

Figure 6. Inhibitory effects of biochanin A on the phosphorylation of c-Jun NH2-terminal kinase (JNK), extracellular signal-regulated kinase (ERK) and p38 in BV2 cells. BV2 cells were pre-incubated with or without biochanin A for $30 \mathrm{~min}$ and then stimulated with lipopolysaccharide (LPS; $10 \mu \mathrm{g} / \mathrm{ml}$ ) for $36 \mathrm{~h}$. The expression levels of (A) p-JNK, (B) p-ERK and (C) p-p38 were measured by western blot analysis. JNK, ERK and p38 were used as internal controls. Results are presented as the means \pm SD of at least 3 independent experiments. ${ }^{* *} \mathrm{P}<0.01$ compared with the control group; ${ }^{*} \mathrm{P}<0.05$ compared with the LPS-treated group. 
demonstrated that biochanin A inhibited the LPS-induced activation of microglia and the production of TNF- $\alpha$, NO and superoxide in mesencephalic neuron-glia cultures and microglia-enriched cultures (11). However, the mechanisms underlying the anti-inflammatory effects of biochanin A are not yet fully understood. Therefore, in this study, in order to elucidate the molecular mechanisms responsible for the antiinflammatory effects of biochanin A, we further investigated the inhibitory effects of biochanin A on the production of proinflammatory factors induced by LPS in murine BV2 microglial cells. Our results demonstrated that biochanin A significantly inhibited the LPS-induced microglial activation and the release of TNF- $\alpha$, IL-1 $\beta$, NO and ROS. Biochanin A also significantly attenuated the LPS-induced mRNA and protein expression of TNF- $\alpha$, IL-1 $\beta$ and iNOS. Furthermore, biochanin A significantly inhibited the LPS-induced phosphorylation of JNK, ERK and p38 in BV2 microglial cells.

Microglia are the resident immune cells of the brain. In response to injury or infection, microglia become readily activated and consequently release pro-inflammatory cytokines, free radicals and eicosanoids. These factors are believed to contribute to microglia-mediated neurodegeneration $(2,19,20)$. Therefore, the inhibition of microglial activation may prove to be an effective therapeutic approach for alleviating the progression of neurodegenerative diseases, such as PD and Alzheimer's disease. In the present study, our results indicated that biochanin A significantly inhibited the LPS-induced microglial activation and the release of pro-inflammatory factors.

Pro-inflammatory cytokines, such as TNF- $\alpha$ and IL-1 $\beta$, have been shown to induce neuronal cell damage; therefore, the suppression of their production is important for the prevention of neurodegenerative diseases. Microglia are the primary producers of TNF- $\alpha$ in the brain and play a role in a number of pathological conditions in the brain (21). TNF- $\alpha$ overexpression has been implicated in the pathogenesis of several human disorders of the central nervous system (CNS) (22-25). IL-1 $\beta$ is a potent pro-inflammatory cytokine that acts through IL-1 receptors found on numerous cell types, including neurons and microglia. Moreover, the importance of IL-1 $\beta$ as a mediator of neuroimmune interactions that participate directly in neurodegeneration has been demonstrated (26). In this study, our results revealed that the production of the pro-inflammatory cytokines, TNF- $\alpha$ and IL-1 $\beta$, was significantly inhibited by biochanin A in a dose-dependent manner. Moreover, the LPS-stimulated mRNA expression levels of TNF- $\alpha$ and IL-1 $\beta$ were also reduced by biochanin $A$, suggesting that biochanin $A$ suppressed the production of TNF- $\alpha$ and IL-1 $\beta$ through the downregulation of their gene expression in the LPS-stimulated microglial cells.

In mammalian cells, $\mathrm{NO}$ is synthesized from three different isoforms of NOS: endothelial NOS (eNOS), neuronal NOS (nNOS) and iNOS. Activated microglia are a major cellular source of iNOS in the brain. The excessive release of NO by activated microglia correlates with the progression of neurodegenerative disorders. The excessive accumulation of NO has long been known to be toxic to neurons $(8,27,28)$. The generation of NO has been implicated in a variety of neuroinflammatory and neurodegenerative disorders. In the brain, iNOS is the main contributor to NO production following an inflammatory assault, compared with the other isoforms of NOS $(29,30)$.
Accumulating evidence indicates that the expression of iNOS during inflammation in the CNS plays an important role in the neurodegeneration in PD (31). It has been reported that activated microglia cause neuronal death with mechanisms involving the expression of iNOS and the release of NO in mixed neuronglia co-cultures (19). This study also demonstrated that LPS induced the activation of microglia, which subsequently led to the upregulation of NO production and iNOS expression. However, pre-treatment with biochanin A exerted a significant inhibitory effect on the LPS-induced the production of NO and attenuated the expression of iNOS in a dose-dependent manner.

Previous studies have suggested that LPS increases the levels of intracellular ROS, which serve as second messengers to enhance the LPS-induced expression of genes encoding a variety of pro-inflammatory factors $(32,33)$. ROS, including superoxide anion, hydroxyl radical, lipid hydroperoxides and their byproducts (e.g., hydrogen peroxide), may play an important role in the pathogenesis of neurodegenerative diseases. ROS generated by activated microglia are toxic to neurons by inducing lipid peroxidation, DNA fragmentation and protein oxidation (34). Moreover, oxygen free radicals, such as superoxide can react with NO to form much more deadly intermediates, such as peroxynitrite (35). In fact, a previous study identified peroxynitrite as a key mediator of neurotoxicity induced by LPS-activated microglia (36). In this study, our results demonstrated that LPS increased intracellular ROS production in BV2 cells. However, pre-treatment with biochanin A attenuated the LPS-induced intracellular ROS production in a dose-dependent manner.

The mammalian MAPK family consists of ERK, p38, and JNK (37). Previous studies have demonstrated that MAPKs are involved in the regulation of iNOS, COX-2, TNF- $\alpha$, IL-1 $\beta$ expression in microglia (38-42). In this study, we found that biochanin A inhibited the LPS-induced phosphorylation of JNK, ERK and p38. In addition, we found that biochanin A inhibited the gene expression of iNOS, TNF- $\alpha$ and IL-1 $\beta$ in LPS-stimulated BV2 microglia, the mechanisms of which at least in part, may involve the inhibition of MAPKs. Taken together, the results from the present study suggest that the inhibition of the MAPK pathway may be a molecular mechanism underlying the anti-inflammatory effects of biochanin A in LPS-stimulated BV2 microglial cells.

In conclusion, to the best of our knowledge, in this study, we demonstrate for the first time that biochanin A markedly attenuates LPS-induced microglial activation and pro-inflammatory responses in BV2 microglial cells. Biochanin A also inhibited the production of IL-1 $\beta, \mathrm{TNF}-\alpha$, NO and ROS, which may be mediated through the inhibition of MAPK signaling pathways in BV2 cells. These observations suggested that biochanin A may be considered as a potential anti-inflammatory agent. However, further in vivo investigations using animal models are required to confirm the effectiveness of biochanin $\mathrm{A}$.

\section{Acknowledgements}

This study was supported by the National Natural Science Foundation of China (NSFC) fund (grant no. 31171650), the National Basic Research Program of China (973 program, no. 2012CB525003) and the Natural Science Foundation of Anhui Province Education Department (Grant no. KJ2014A115). 


\section{References}

1. Liu B and Hong JS: Role of microglia in inflammation-mediated neurodegenerative diseases: mechanisms and strategies for therapeutic intervention. J Pharmacol Exp Ther 304: 1-7, 2003.

2. McGeer PL, Itagaki S, Boyes BE and McGeer EG: Reactive microglia are positive for HLA-DR in the substantia nigra of Parkinson's and Alzheimer's disease brains. Neurology 38 : 1285-1291, 1988

3. Kreutzberg GW: Microglia: a sensor for pathological events in the CNS. Trends Neurosci 19: 312-318, 1996.

4. Gao HM, Hong JS, Zhang W and Liu B: Distinct role for microglia in rotenone-induced degeneration of dopaminergic neurons. J Neurosci 22: 782-790, 2002.

5. Gayle DA, Ling Z, Tong C, Landers T, Lipton JW and Carvey PM: Lipopolysaccharide (LPS)-induced dopamine cell loss in culture: roles of tumor necrosis factor-alpha, interleukin-1beta, and nitric oxide. Brain Res Dev Brain Res 133: 27-35, 2002.

6. Kim WG, Mohney RP, Wilson B, Jeohn GH, Liu B and Hong JS: Regional difference in susceptibility to lipopolysaccharideinduced neurotoxicity in the rat brain: role of microglia. J Neurosci 20: 6309-6316, 2000.

7. Liu B, Du L and Hong JS: Naloxone protects rat dopaminergic neurons against inflammatory damage through inhibition of microglia activation and superoxide generation. J Pharmacol Exp Ther 293: 607-617, 2000

8. Liu B, Gao HM, Wang JY, Jeohn GH, Cooper CL and Hon JS: Role of nitric oxide in inflammation-mediated neurodegeneration. Ann NY Acad Sci 962: 318-331, 2002.

9. Jung WK, Ahn YW, Lee SH, Choi YH, Kim SK, Yea SS, Choi I, Park SG, Seo SK, Lee SW and Choi IW: Ecklonia cava ethanolic extracts inhibit lipopolysaccharide-induced cyclooxygenase-2 and inducible nitric oxide synthase expression in BV2 microglia via the MAP kinase and NF-kappaB pathways. Food Chem Toxicol 47: 410-417, 2009.

10. Lei M, Wang JG, Xiao DM, Fan M, Wang DP, Xiong JY, Chen Y Ding Y and Liu SL: Resveratrol inhibits interleukin $1 \beta$-mediated inducible nitric oxide synthase expression in articular chondrocytes by activating SIRT1 and thereby suppressing nuclear factor- $\kappa$ B activity. Eur J Pharmacol 674: 73-79, 2012.

11. Chen HQ, Jin ZY and Li GH: Biochanin A protects dopaminergic neurons against lipopolysaccharide-induced damage through inhibition of microglia activation and proinflammatory factors generation. Neurosci Lett 417: 112-117, 2007.

12. Puthli A, Tiwari R and Mishra KP: Biochanin A enhances the radiotoxicity in colon tumor cells in vitro. J Environ Pathol Toxicol Oncol 32: 189-203, 2013.

13. Su SJ, Yeh YT and Shyu HW: The preventive effect of biochanin a on bone loss in ovariectomized rats: involvement in regulation of growth and activity of osteoblasts and osteoclasts. Evid Based Complement Alternat Med 2013: 594857, 2013.

14. Green LC, Wagner DA, Glogowski J, Skipper PL, Wishnok JS and Tannenbaum SR: Analysis of nitrate, nitrite, and [15N]nitrate in biological fluids. Anal Biochem 126: 131-138, 1982.

15. Bruce-Keller AJ, Keeling JL, Keller JN, Huang FF, Camondola S and Mattson MP: Antiinflammatory effects of estrogen on microglial activation. Endocrinology 141: 3646-3656, 2000.

16. Boje KM: Nitric oxide neurotoxicity in neurodegenerative diseases. Front Biosci 9: 763-776, 2004.

17. Han IO, Kim KW, Ryu JH and Kim WK: p38 mitogen-activated proteinkinase mediates lipopolysaccharide, notinterferon-gamma, -induced inducible nitric oxide synthase expression in mouse BV2 microglial cells. Neurosci Lett 325: 9-12, 2002.

18. Kim M, Li YX, Dewapriya P, Ryu B and Kim SK: Floridoside suppresses pro-inflammatory responses by blocking MAPK signaling in activated microglia. BMB Rep 46: 398-403, 2013.

19. Le W, Rowe D, Xie W, Ortiz I, He Y and Appel SH: Microglial activation and dopaminergic cell injury: an in vitro model relevant to Parkinson's disease. J Neurosci 21: 8447-8455, 2001.

20. Minghetti L and Levi G: Microglia as effector cells in brain damage and repair: focus on prostanoids and nitric oxide. Prog Neurobiol 54: 99-125, 1998.

21. Sawada M, Kondo N, Suzumura A and Marunouchi T: Production of tumor necrosis factor-alpha by microglia and astrocytes in culture. Brain Res 491: 394-397, 1989.
22. Akassoglou K, Bauer J, Kassiotis G, et al: Oligodendrocyte apoptosis and primary demyelination induced by local TNF/ p55TNF receptor signaling in the central nervous system of transgenic mice: models for multiple sclerosis with primary oligodendrogliopathy. Am J Pathol 153: 801-813, 1998.

23. Botchkina GI, Meistrell ME III, Botchkina IL and Tracey KJ: Expression of TNF and TNF receptors (p55 and p75) in the rat brain after focal cerebral ischemia. Mol Med 3: 765-781, 1997.

24. Meda L, Cassatella MA, Szendrei GI, et al: Activation of microglial cells by beta-amyloid protein and interferon-gamma. Nature 374: 647-650, 1995.

25. Sriram K, Matheson JM, Benkovic SA, Miller DB, Luster MI and O'Callaghan JP: Mice deficient in TNF receptors are protected against dopaminergic neurotoxicity: implications for Parkinson's disease. FASEB J 16: 1474-1476, 2002.

26. Rothwell N, Allan S and Toulmond S: The role of interleukin 1 in acute neurodegeneration and stroke: pathophysiological and therapeutic implications. J Clin Invest 100: 2648-2652, 1997.

27. Chao CC, Hu S, Molitor TW, Shaskan EG and Peterson PK: Activated microglia mediate neuronal cell injury via a nitric oxide mechanism. J Immunol 149: 2736-2741, 1992.

28. Jeohn GH, Kim WG and Hong JS: Time dependency of the action of nitric oxide in lipopolysaccharide-interferon-gamma-induced neuronal cell death in murine primary neuron-glia co-cultures. Brain Res 880: 173-177, 2000.

29. Iravani MM, Kashefi K, Mander P, Rose S and Jenner P: Involvement of inducible nitric oxide synthase in inflammationinduced dopaminergic neurodegeneration. Neuroscience 110: 49-58, 2002.

30. Li FQ, Wang T, Pei Z, Liu B and Hong JS: Inhibition of microglial activation by the herbal flavonoid baicalein attenuates inflammation-mediated degeneration of dopaminergic neurons. J Neural Transm 112: 331-347, 2005.

31. Dawson VL and Dawson TM: Nitric oxide in neuronal degeneration. Proc Soc Exp Biol Med 211: 33-40, 1996.

32. Qin L, Liu Y, Wang T, et al: NADPH oxidase mediates lipopolysaccharide-induced neurotoxicity and proinflammatory gene expression in activated microglia. J Biol Chem 279: 1415-1421, 2004.

33. Wang T, Qin L, Liu B, et al: Role of reactive oxygen species in LPS-induced production of prostaglandin E2 in microglia. J Neurochem 88: 939-947, 2004.

34. Farber JL: Mechanisms of cell injury by activated oxygen species. Environ Health Perspect 102 (Suppl 10): S17-S24, 1994.

35. Estevez AG and Jordan J: Nitric oxide and superoxide, a deadly cocktail. Ann NY Acad Sci 962: 207-211, 2002.

36. Xie Z, Wei M, Morgan TE, et al: Peroxynitrite mediates neurotoxicity of amyloid beta-peptide1-42- and lipopolysaccharide-activated microglia. J Neurosci 22: 3484-3492, 2002.

37. Kim EK and Choi EJ: Pathological roles of MAPK signaling pathways in human diseases. Biochim Biophys Acta 1802: 396-405, 2010.

38. Bhat NR, Zhang P, Lee JC and Hogan EL: Extracellular signalregulated kinase and p38 subgroups of mitogen-activated protein kinases regulate inducible nitric oxide synthase and tumor necrosis factor-alpha gene expression in endotoxin-stimulated primary glial cultures. J Neurosci 18: 1633-1641, 1998.

39. Choi Y, Lee MK, Lim SY, Sung SH and Kim YC: Inhibition of inducible NO synthase, cyclooxygenase-2 and interleukin-1beta by torilin is mediated by mitogen-activated protein kinases in microglial BV2 cells. Br J Pharmacol 156: 933-940, 2009.

40. Johnson GL and Lapadat R: Mitogen-activated protein kinase pathways mediated by ERK, JNK, and p38 protein kinases. Science 298: 1911-1912, 2002.

41. Park JS, Woo MS, Kim DH, et al: Anti-inflammatory mechanisms of isoflavone metabolites in lipopolysaccharide-stimulated microglial cells. J Pharmacol Exp Ther 320: 1237-1245, 2007.

42. Song Y, Qu R, Zhu S, Zhang R and Ma S: Rhynchophylline attenuates LPS-induced pro-inflammatory responses through downregulation of MAPK/NF-kappaB signaling pathways in primary microglia. Phytother Res 26: 1528-1533, 2012. 